\title{
Imagens de alta resolução na análise da acessibilidade para cadeirantes na Universidade Federal de Pernambuco - UFPE
}

High resolution images in the analysis of accessibility for wheelchair at the Federal University of Pernambuco - UFPE

\author{
Elaine Cristina Osorio Rocha ${ }^{1}$ \\ Ariely Mayara de Albuquerque Teixeira² \\ Marianny Monteiro Pereira de Lira ${ }^{3}$ \\ Ana Lúcia Bezerra Candeias ${ }^{4}$ \\ Lucilene Antunes Correia Marques de Sá ${ }^{5}$
}

\begin{abstract}
1, 2, 4, 5 Departamento de Engenharia Cartográfica, Universidade Federal de Pernambuco - UFPE.
Av. Prof. Moraes Rego, 1235 - Cidade Universitária, Recife - PE. CEP 50670-901.

elainecris@ifpi.edu.br, ariely.albuq@gmail.com, analucia@ufpe.br, lacms@ufpe.br
\end{abstract}

${ }^{3}$ Departamento de Engenharia Civil, Universidade Federal de Pernambuco - UFPE.

Av. Prof. Moraes Rego, 1235 - Cidade Universitária, Recife - PE. CEP 50670-901.

marianny.monteiro@gmail.com

Recebido em 19/12/2015 - Aceito em 09/12/2016

Received on 19/12/2015 - Accepted on 09/12/2016

\begin{abstract}
RESUMO - Neste estudo buscou-se identificar e avaliar a qualidade dos acessos específicos para mobilidade e acessibilidade de cadeirantes nos setores de atendimento à saúde para o público interno e externo da UFPE utilizando imagens de alta resolução disponíveis no Google Earth Pro comparando com a legislação específica. Para identificação de rampas e calçadas rebaixadas, foram georreferenciadas com GPS de navegação e complementada com levantamento fotográfico. Foram realizadas aferições com trena para análise qualitativa de acordo com a NBR 9050/2004. A pesquisa propõe uma classificação para avaliar a condição física da superfície dos acessos indicando se os parâmetros estão em conformidade com a Norma. Pela distribuição espacial usando imagens de sensoriamento remoto associados com os dados de campo, pode-se perceber que há número considerável de acessos destinados a mobilidade de pessoas em cadeiras de rodas na UFPE mas que necessitam de melhorias das condições físicas das calçadas, rampas, calçadas rebaixadas e sinalização indicativa uma vez que a área é utilizada também para tratamento de pessoas com deficiência e mobilidade reduzida e necessitam de acesso seguro e autônomo aos serviços ofertados pela Universidade.
\end{abstract}

Palavras-chave: Sensoriamento Remoto, GPS de Navegação, Acessibilidade para Cadeirantes

\begin{abstract}
In this study we sought to identify and assess the quality of specific access to mobility and wheelchair accessibility in the health care sectors for the internal and external public UFPE using high-resolution images available on Google Earth Pro comparing with specific legislation. For identification ramps and lowered sidewalks were georeferenced with GPS navigation and completed with photographic survey. Measurements were performed with measuring tape to qualitative analysis according to NBR 9050/2004. The research proposes a classification to assess the physical condition of the surface of accesses indicating whether the parameters are in accordance with the Standard. The spatial distribution using remote sensing images associated with field data, it can be seen that there is considerable number of hits for the mobility of people in wheelchairs at university but for improvement of the physical condition of the sidewalks, ramps, lowered sidewalks and signs indicating since the area is also used to treat people with disabilities and reduced mobility and require safe and autonomous access to the services offered by the University
\end{abstract}

Keywords: Remote Sensing, GPS Navigation, Accessibility for Wheelchair users

\section{INTRODUÇÃO}

De forma ideal, o espaço urbano deve ser plenamente acessível a todos e para isso, todos os elementos físicos e equipamentos urbanos devem estar adaptados conforme base normativa e conectados entre si para permitir mobilidade e fluidez na locomoção de pessoas com deficiências.
A falta de mobilidade e acessibilidade nos espaços urbanos geram dificuldades de locomoção e, os obstáculos físicos das vias constituem um desafio diário na tarefa de ir e vir dificultando o acesso aos serviços e locais pelos cidadãos. Isto se torna mais visível para pessoas em cadeiras de rodas.

Os resultados do Censo Demográfico de 2010 revelaram que 45.606.048 milhões de pessoas declararam 
ter pelo menos um tipo de deficiência. A região Nordeste concentra os maiores percentuais da população com, pelo menos, um tipo de deficiência $(26,6 \%)$. O Estado de Pernambuco ocupa a $2^{\mathrm{a}}$ posição no ranking Nacional de pessoas com deficiência motora. A cidade de Recife ocupa a $2^{a}$ posição entre as capitais brasileiras, com $19 \%$ da população em domicílio urbano de pessoas com deficiência motora.

Nos espaços de circulação das universidades a situação não é diferente. Os obstáculos arquitetônicos como também a inexistência de rampas, rampas inadequadas, elevadores que não funcionam, calçadas em mau estado de conservação e sem sinalização impedem a locomoção de forma segura e autônoma por alunos, servidores e o público externo de executarem tarefas como assistir aulas, trabalhar e terem acesso aos diversos serviços ofertados.

No Brasil, a política de inclusão social das pessoas com deficiência existe desde a Constituição de 1988, que originou a Lei $n^{\circ}$. 7.853/1989, posteriormente regulamentada pelo Decreto $\mathrm{n}^{\circ}$. 3.298/1999. Esses documentos nacionais, junto as Leis $\mathrm{n}^{\circ}$. 10.048, $\mathrm{n}^{\circ} .10 .098$, de 2000, Decreto $\mathrm{n}^{\circ}$. 5.296/2004 conhecido como o Decreto da Acessibilidade e a ABNT no 9050/2004 regulamentam o acesso de pessoas com deficiências aos espaços públicos e privados. Os espaços de circulação devem ser adaptados conforme descrito na Norma da ABNT no 9050/2004 que estabelece critérios e parâmetros técnicos para acessibilidade a edificações, mobiliário, espaços e equipamentos urbanos.

A partir da identificação dos elementos físicos urbanos destinados a pessoas em cadeiras de rodas é possível fornecer procedimentos para intervenção e adequação dos espaços além de análises de rotas para auxiliar na locomoção. Para isso, Jensen (2009) e Vasques (2009) afirmam que diversos setores utilizam cada vez mais as geotecnologias para análises urbanas. O autor complementa que a identificação dos alvos a estudar pode variar de acordo com a escala, pois a mesma determina o nível de detalhe da informação.

Para Cardoso et al. (2014) e Cardoso (2015) a elaboração de mapas de acessibilidade é uma ferramenta eficaz para espacializar os elementos físicos do espaço urbano que interferem na acessibilidade, identificam áreas problemáticas e grau de acessibilidade auxiliando na escolha de pontos e rotas para deslocamento através da visualização nos mapas.

Goodchild (2008) e Goodchild (2012) analisaram as aplicações das geoinformações a partir de geobrowsers ou geoportais. Também em Longley et al. (2016) tem-se uma apresentação dos Sistemas de Informação Geográfica, mostrando seus princípios, técnicas, gestão e aplicações. Com o auxílio das Tecnologias da Geoinformação, e também do Sensoriamento Remoto e do Posicionamento por GNSS é possível se fazer a identificação, a representação e a localização geográfica de alvos a partir de imagens de satélites, dos pontos de controle e dos mapas temáticos, e possibilitam desenvolver diversas metodologias para compreender o espaço.
Nesse artigo apresenta-se um método para auxiliar na identificação dos elementos físicos para acessibilidade e para a mobilidade de pessoas em cadeiras de rodas a partir de imagens de alta resolução espacial. A área escolhida é a Universidade Federal de Pernambuco (UFPE). Para isso foram utilizadas imagens disponíveis do Google Earth Pro e pontos de controle coletados a partir de GPS de navegação. A pesquisa gerou como resultado, a avaliação qualitativa de rampas e calçadas rebaixadas a partir de uma classificação baseada nas características físicas e na inclinação com base na legislação NBR 9050/2004, além, de fornecer a distribuição espacial dos acessos em imagem de satélite em um trecho da UFPE.

\section{REFERENCIAL TEÓRICO}

Os termos mobilidade e acessibilidade estão correlacionados, já que mobilidade é a facilidade de deslocamento e acessibilidade são as formas e oportunidades oferecidas no espaço urbano de acesso a determinado local, e são dependentes, pois segundo Aguiar (2008) quando se aumenta o nível de acessibilidade a determinado espaço, espera-se aumentar também as condições de mobilidade oferecidas aos seus usuários.

Leite (2013) declara que a mobilidade em seu sentido mais amplo remete à acessibilidade às localidades, ou seja, a acessibilidade não é apenas a facilidade de circular no território, mas a facilidade de chegar aos destinos.

Aguiar (2008) complementa que a mobilidade depende do desempenho do espaço e das características do próprio indivíduo (capacidade de locomoção). Deve-se avaliar as condições de acessibilidade dos espaços destinados para pessoas com deficiência para facilitar a locomoção e oferecer subsídios para intervenções urbanísticas.

Vasconcellos (2001) preconiza que a avaliação da acessibilidade pode ser uma medida similar direta à densidade das linhas de transporte público, densidade das vias e avaliação em relação ao tempo. Nessa perspectiva, o conceito de acessibilidade pode estender-se a uma relação direta com a densidade de vias de acessos e rampas que possibilitam de forma segura acesso aos espaços públicos e serviços.

Araújo (2005) afirma que as normas que regem o padrão construtivo das rampas devem ser destacadas, uma vez que, apenas a existência desta não garante a pessoa com deficiência motora deslocar-se livremente.

A norma da ABNT NBR 9050/2004 estabelece critérios e parâmetros técnicos para adaptação dos espaços físicos destinados a pessoas em cadeiras de rodas, assim descritos:

a) A largura mínima da rampa deve ser de 1,20m, contendo ainda rampas laterais de no mínimo $0,50 \mathrm{~m}$ junto ao meio-fio, como mostra a Figura 18. A declividade não deve exceder a $12,5 \%$ (1:8). O objetivo é garantir que a faixa de circulação seja plana, livre e contínua ao passeio público em frente à rampa de $0,80 \mathrm{~m}$ de largura. Caso contrário deve haver rebaixamento em todo o passeio público com rampas 
cuja declividade tem que ser inferior a 12,5\% (Figura 1).

b) Conforme o caso, o rebaixamento das calçadas pode apresentar diferentes configurações:

- Rebaixamento total da calçada na esquina;

- Rebaixamento total da largura da calçada, com largura mínima de $1,50 \mathrm{~m}$ e com rampas laterais com inclinação máxima de $8,33 \%$ (quando a largura do passeio não for suficiente para acomodar o rebaixamento e a faixa livre);

- Rebaixamento, de no mínimo $0,80 \mathrm{~m}$, sendo recomendável 1,20m (para faixa livre de passeio, além do espaço ocupado pelo rebaixamento).

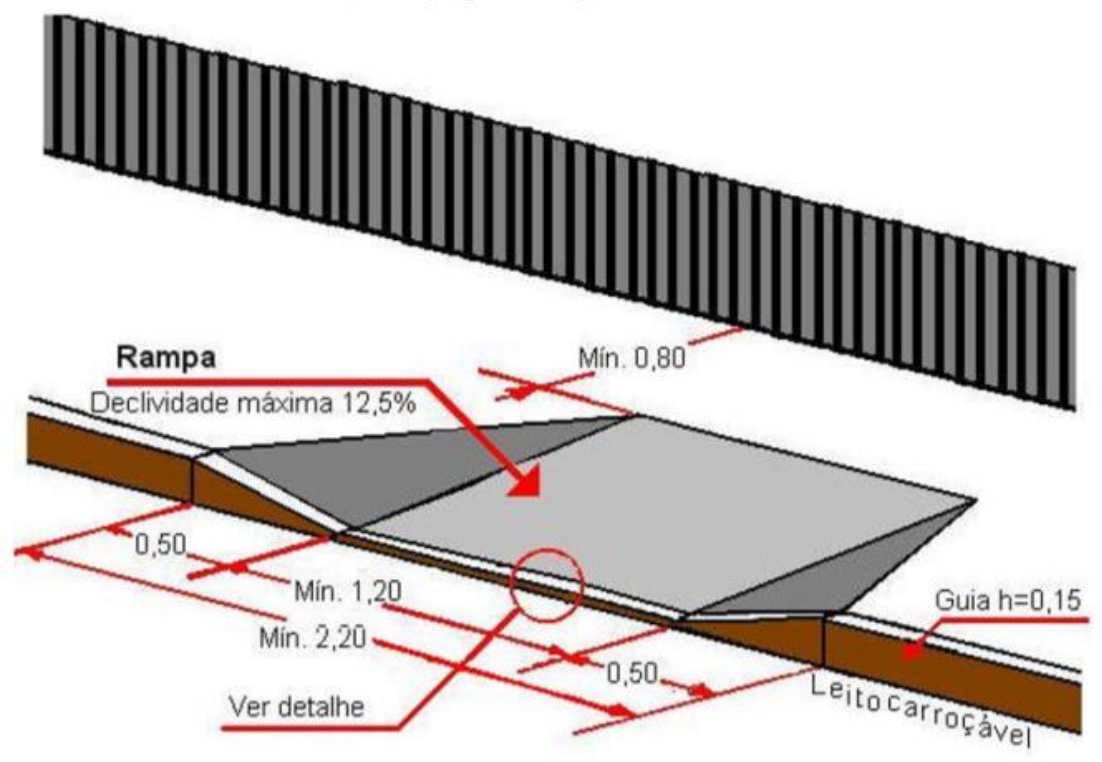

Figura 1 - Dimensões das rampas Fonte: NBR 9050/2004.

Pela NBR 9050/2004, calçada rebaixada é rampa construída ou implantada na calçada ou passeio, destinada a promover a concordância de nível entre estes e o leito carroçável.

Pisos devem apresentar superfície regular, continua, sem ressalto ou depressão, firme, estável, antiderrapante (sob quaisquer condições climáticas), de forma a não provocar trepidação em dispositivos com rodas.

A inclinação máxima em rampa recomendada para vias de pedestres é de 8,33\% (para rotas acessíveis). Inclinações até $12,5 \%$ são utilizadas com restrições, mas não fazem parte de rotas acessíveis. A inclinação de rampas é calculada pela Equação 1:

Onde:

$$
I=(h / c)^{*} 100 .
$$

$$
\begin{aligned}
& \text { I = inclinação } \\
& h=\text { desnível } \\
& c=\text { comprimento }
\end{aligned}
$$

Velasco (2007) declara que a utilização das imagens de satélites em ambiente urbano tornou uma prática bastante utilizada. As imagens de alta resolução espacial permitem identificar com maior facilidade todos os componentes que constituem o espaço urbano. Jensen (2009), Vasques (2009) complementam que a disponibilidade de imagens de alta resolução para estudos sobre as áreas urbanas possui uma contribuição inegável, seja para a cartografia, o monitoramento ou $o$ planejamento.

Segundo Jensen (2009), o Sensoriamento Remoto aliado à Cartografia auxiliam nas representações espaciais e planejamento do território além da implantação de infraestruturas urbanas.

\section{3. ÁREA DE ESTUDO}

A área de estudo escolhida foi o espaço onde estão localizados os setores que oferecem atendimento à saúde para o público interno e externo da UFPE, incluindo atendimento para pessoas com deficiência e mobilidade reduzida, e com um número significativo de vias de acesso. A Figura 2 apresenta o trecho escolhido para o levantamento. 

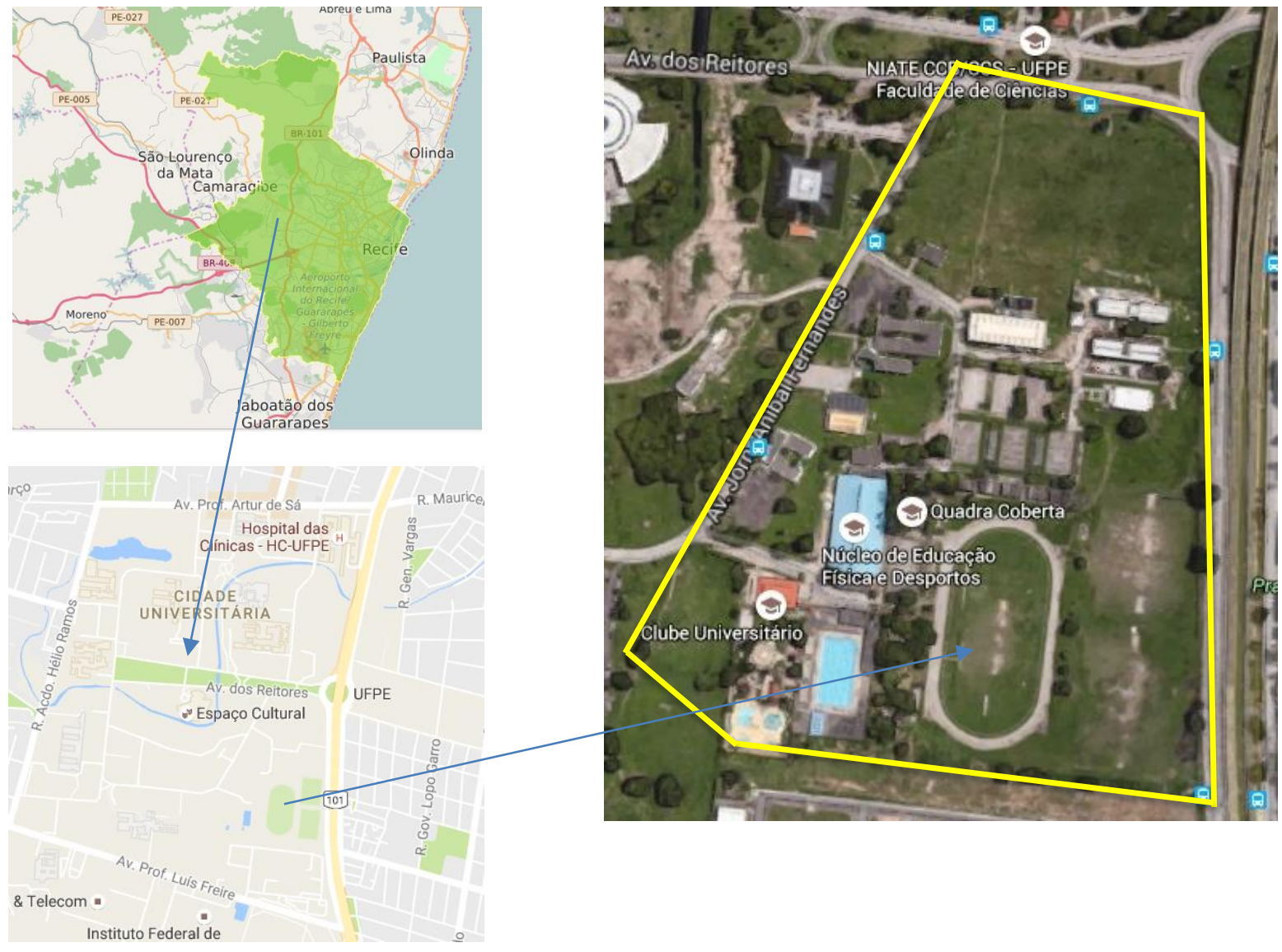

Figura 2 - Croqui do trecho da UFPE escolhido para o levantamento. Adaptado: IBGE (2016), Google Maps e Google Earth Pro.

\section{PROCEDIMENTO METODOLÓGICO}

O uso do GPS de navegação foi para a identificação dos elementos físicos para acessibilidade de cadeirantes para suporte no deslocamento de pessoas em cadeiras de rodas. Portanto, a etapa inicial compreendeu do levantamento de pontos de controle (rampas e calçadas rebaixadas) com uso de GPS de navegação, marca Garmin, modelo Etrex, rastreamento código $\mathrm{CA}$, precisão aproximada $5 \mathrm{~m}$. Foram adquiridos 10 pontos de controle compreendendo o Clube Universitário, o Centro de Educação Física, os prédios de Terapia Ocupacional (T.O) e Fisioterapia, como mostra a Figura 2 e as coordenadas descritas na Tabela 1.

Também foram realizados registros fotográficos de cada tipo de acesso coletado em campo para análise das características físicas e aferição com trena para cálculo da inclinação para avaliar o comprimento da norma NBR 9050/2004 (Figura 3).

As informações sobre as condições físicas dos acessos e valores de inclinação foram reunidos na Tabela 1 e cada ponto recebeu uma classificação. A pesquisa atribui indicadores para facilitar a escolha dos acessos para locomoção por parte das pessoas em cadeiras de rodas conforme grau de acessibilidade.
Tabela 1 - Pontos de controle do campo

\begin{tabular}{c|c|c|l}
\hline Ponto & $\mathbf{E}(\mathbf{m})$ & $\mathbf{N}(\mathbf{m})$ & \multicolumn{1}{|c}{ Observação } \\
\hline 1 & 285123 & 9109124 & Rampa de acesso. \\
\hline 2 & 285135 & 9109145 & $\begin{array}{l}\text { Rampa de acesso ao } \\
\text { prédio de T. O. }\end{array}$ \\
\hline 3 & 285143 & 9109145 & $\begin{array}{l}\text { Rampa de acesso ao } \\
\text { prédio. }\end{array}$ \\
\hline 4 & 285199 & 9109145 & $\begin{array}{l}\text { Rampa de acesso a } \\
\text { lanchonete da quadra. }\end{array}$ \\
\hline 5 & 285272 & 9109110 & $\begin{array}{l}\text { Rampa de acesso ao } \\
\text { campo de futebol. }\end{array}$ \\
\hline 6 & 285229 & 9109001 & $\begin{array}{l}\text { Rampa de acesso à } \\
\text { piscina. }\end{array}$ \\
\hline 7 & 285226 & 9109035 & $\begin{array}{l}\text { Rampa de acesso ao } \\
\text { estacionamento. }\end{array}$ \\
\hline 9 & 285164 & 9109193 & Rampa ao lado T.O. \\
\hline 10 & 285225 & 9109231 & $\begin{array}{l}\text { Rampa no prédio de } \\
\text { fisioterapia. }\end{array}$ \\
\hline
\end{tabular}

Os pontos de controle coletados em campo foram importados para Google Earth Pro determinando a localização espacial das rampas e calçadas rebaixadas. $\mathrm{O}$ 
Google Earth Pro foi associado ao Street View para análise visual dos acessos. Foi utilizado imagem disponível gratuitamente no Google Earth Pro datada de 26 de janeiro de 2007 pois oferecia melhor visualização da área de estudo (Figura 3). Com o Google Earth Pro pode-se armazenar e atualizar informações de texto, fotos entre outros. O Google Earth é um geovisualizador de simples utilização para o público em geral, além de ser robusto do ponto de vista cartográfico e pode auxiliar na aplicação proposta.

\section{RESULTADOS E DISCUSSÃO}

$\mathrm{Na}$ avaliação qualitativa dos elementos físicos para mobilidade e acessibilidade na área de estudo, observou-se a falta de sinalização indicativa de rampas nos espaços percorridos para auxílio na identificação visual para usuários de cadeiras de rodas.

As calçadas apresentaram piso irregular, há trechos quebrados, desnivelados e rachados, como indicado nas fotos dos pontos 02, 07 e 09 (Figura 3). Não existe o respeito com relação do padrão construtivo das calçadas definido na NBR 9050/2004 que deve apresentar superfície regular, contínua, sem ressalto ou depressão.

Em alguns locais percorridos foram identificados diferentes tipos de materiais do piso ao longo do mesmo trecho. Os tipos de materiais encontrados nas calçadas foram cimento e cerâmica antiderrapante. Mesmo sendo indicados pela NBR 9050/2004 por não causar trepidação nas cadeiras de rodas, existem locais em que o piso está em mau estado de conservação e isso dificulta a mobilidade, como indicam as fotos dos pontos 07 e 09.
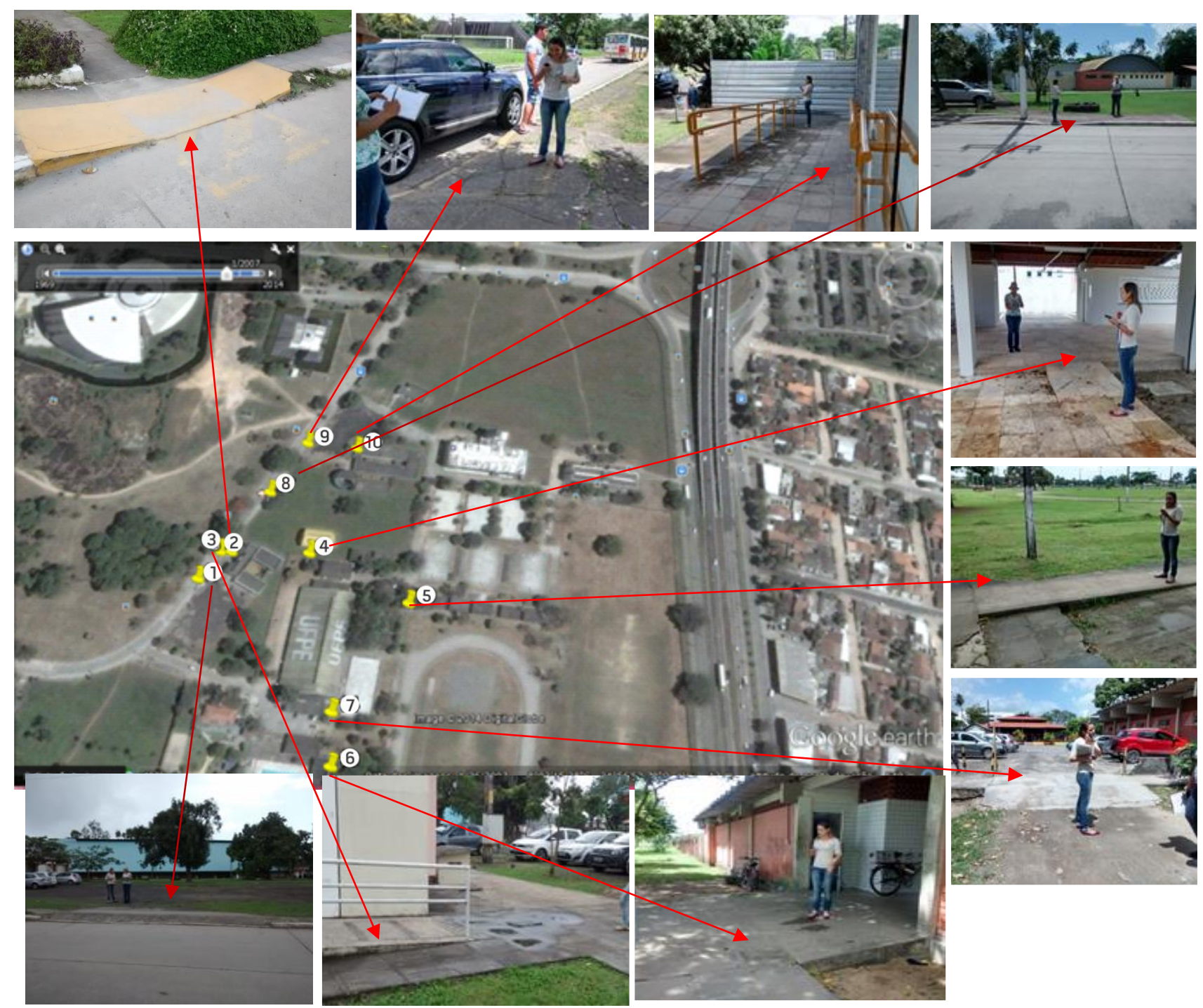

Figura 3 - Imagem do Google Earth Pro com o registro fotográfico para as rampas e calçadas rebaixadas.

$\mathrm{Na}$ área estudada, existem obstáculos físicos ao longo das calçadas, como postes e árvores, que impossibilitam o deslocamento por pessoas em cadeiras de rodas, como mostra a foto do ponto 8 (Figura 3) e foto 1 (Figura 4).
A calçada rebaixada apresentada na foto 07 (Figura 4) mostra um desnível em relação ao trecho seguinte. A má qualidade do trecho é um obstáculo para a mobilidade de forma segura e autônoma de usuários de cadeiras de rodas. 
Pela avaliação conjunta das características físicas das calçadas, rampas e calçadas rebaixadas a partir do levantamento fotográfico, conclui-se que a falta de padronização quanto a condição física dos elementos para acessibilidade é um fator crucial de impedimento à mobilidade e acesso aos prédios que prestam serviços de saúde e setor de esportes para pessoas em cadeiras de rodas na UFPE.
A Figura 4 apresenta as dimensões das rampas e calçadas rebaixadas da área de estudo. A Tabela 2 reune as características físicas e os valores da inclinação conforme Equação 1, indicando se estão ou não conforme recomendação da NBR 9050/2004. Apenas 04 acessos, do total de 10 (dez) possuem inclinação indicada na norma. A Tabela 2 indica que mesmo os acessos com inclinação adequada apresentam os pisos fora do padrão recomendado
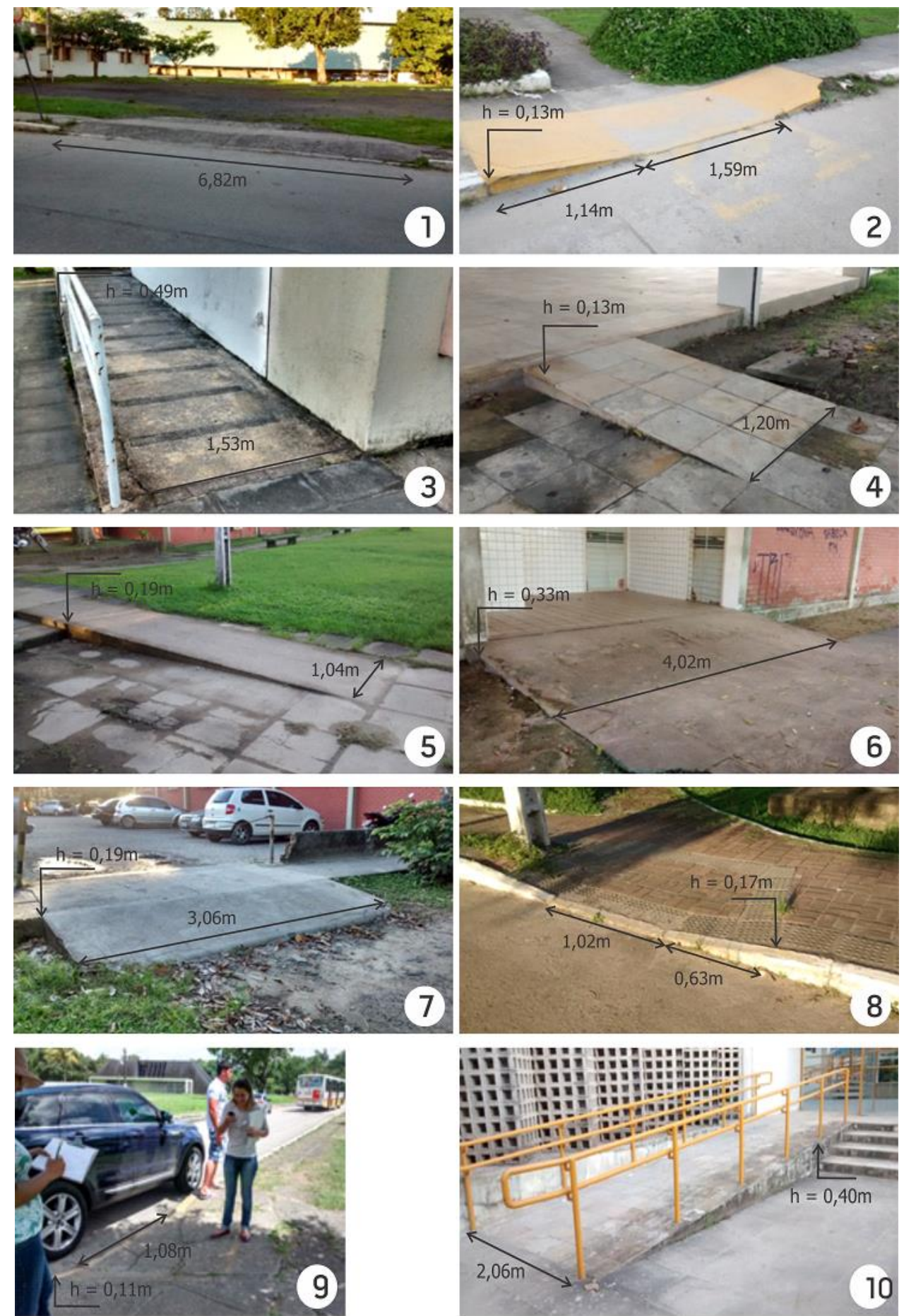

Figura 4 - Levantamento fotográfico das rampas e calçadas rebaixadas aferidas com trena. 
Tabela 2 - Análise qualitativa dos acessos segunda NBR 9050/2004

\begin{tabular}{|c|c|c|c|c|c|c|c|}
\hline Ponto & Tipo & $\begin{array}{l}\text { Desnível } \\
\text { (m) }\end{array}$ & $\begin{array}{c}\text { Comprimento } \\
(\mathbf{m})\end{array}$ & $\begin{array}{c}\text { Inclinação } \\
(\%)\end{array}$ & $\begin{array}{l}\text { Condição } \\
\text { Superfície }\end{array}$ & $\begin{array}{c}\text { Classificação } \\
\text { (Acessível) }\end{array}$ & Observação \\
\hline 1 & $\begin{array}{l}\text { Calçada } \\
\text { rebaixada }\end{array}$ & 0,13 & 6,82 & 1,90 & $\begin{array}{l}\text { Rachada } \\
\text { Irregular }\end{array}$ & Moderada & $\begin{array}{c}\text { Inclinação conforme NBR } \\
9050 / 2004 \\
(\text { até } 8,33 \%)\end{array}$ \\
\hline 2 & $\begin{array}{l}\text { Calçada } \\
\text { rebaixada }\end{array}$ & 0,13 & 1,59 & 8,17 & Boa & Alta & $\begin{array}{c}\text { Inclinação conforme NBR } \\
9050 / 2004 \\
(\text { até } 8,33 \%)\end{array}$ \\
\hline 3 & Rampa & 0,49 & 1,53 & 32 & Rachada & Muito baixa & $\begin{array}{l}\text { Inclinação acima do } \\
\text { recomendado (até 12,5\%) }\end{array}$ \\
\hline 4 & Rampa & 0,13 & 1,20 & 10,8 & Boa & Baixa & $\begin{array}{c}\text { Inclinação acima do } \\
\text { recomendado (até 8,33\%) }\end{array}$ \\
\hline 5 & Rampa & 0,19 & 1,04 & 18,3 & Boa & Muito baixa & $\begin{array}{c}\text { Inclinação acima do } \\
\text { recomendado (até 8,33\%) }\end{array}$ \\
\hline 6 & Rampa & 0,33 & 4,02 & 8,2 & Rachada & Moderada & $\begin{array}{c}\text { Inclinação conforme NBR } \\
9050 / 2004 \\
(\text { até } 8,33 \%)\end{array}$ \\
\hline 7 & Rampa & 0,19 & 3,06 & 6,2 & $\begin{array}{c}\text { Rachada; } \\
\text { Discordância do nível } \\
\text { entre rampa e o } \\
\text { trecho seguinte. } \\
\end{array}$ & Inacessível & $\begin{array}{c}\text { Inclinação conforme NBR } \\
9050 / 2004 \\
(\text { até } 8,33 \%)\end{array}$ \\
\hline 8 & $\begin{array}{l}\text { Calçada } \\
\text { rebaixada }\end{array}$ & 0,17 & 1,2 & 14,2 & Boa & Muito baixa & $\begin{array}{l}\text { Inclinação acima do } \\
\text { recomendado (até 8,33\%) }\end{array}$ \\
\hline 9 & $\begin{array}{l}\text { Calçada } \\
\text { rebaixada }\end{array}$ & 0,11 & 1,08 & 10,2 & Rachada & Baixa & $\begin{array}{l}\text { Inclinação acima do } \\
\text { recomendado (até 8,33\%) }\end{array}$ \\
\hline 10 & Rampa & 0,40 & 2,06 & 19,4 & Boa & Muito baixa & $\begin{array}{l}\text { Inclinação acima do } \\
\text { recomendado (até 12,5\%) }\end{array}$ \\
\hline
\end{tabular}

A classificação dos elementos físicos quanto a mobilidade e acessibilidade de pessoas em cadeiras de rodas foi criada atribuindo indicadores baseados na análise conjunta das condições físicas das rampas e calçadas rebaixadas, da inclinação e autonomia para mobilidade. Os acessos foram classificados assim:

- Acessibilidade Alta - acessos com boa condição físico do piso e inclinação conforme a norma;

- Acessibilidade Moderada - acessos com piso rachados e inclinação conforme a norma;

- Acessibilidade Baixa - acessos com inclinação fora do padrão e locomoção com dificuldade;

- Acessibilidade Muito Baixa - acessos com inclinação fora do padrão e com auxílio de terceiros (acessibilidade assistida).

A análise qualitativa através da classificação indica que somente a calçada rebaixada 2 possui Acessibilidade Alta, dentro dos padrões da Norma NBR 9050/2004 quanto as condições físicas e inclinação.

A calçada rebaixada $n^{\circ} 1$ e a rampa $n^{\circ} 6$ estão classificadas como Acessibilidade Moderada, apresentam o piso rachado, mas não compromete o acesso de forma autônoma.
A rampa $n^{\circ} 4$ e a calçada rebaixada $n^{\circ} 9$ são consideradas como Acessibilidade Baixa, o piso rachado não impossibilita o acesso e os valores da inclinação pouco acima do recomendado permite o uso, de forma autônoma, mas com dificuldade, por pessoas em cadeiras de rodas.

As rampas $n^{\circ} 3,4,10$ e a calçada rebaixada $n^{\circ} 8$ são classificadas como Acessibilidade Muito Baixa pela alta inclinação das mesmas que impossibilitam o acesso de forma segura e autônoma, é possível somente com o auxílio de outra pessoa. Essa forma é chamada de Acessibilidade Assistida.

A rampa $n^{\circ} 7$ apesar de estar classificada como moderada ela torna-se inacessível por conta da falta de piso adequado para mobilidade no trecho seguinte.

A análise qualitativa das rampas e calçadas rebaixadas foi inserida no Google Earth Pro como atributo em cada ponto utilizando as ferramentas do programa e associada ao Street View (Figura 5). Assim, foi possível a visualização da distribuição espacial dos elementos físicos para acessibilidade auxiliando o usuário de cadeira de rodas a escolher o ponto que mais favorece no deslocamento. 


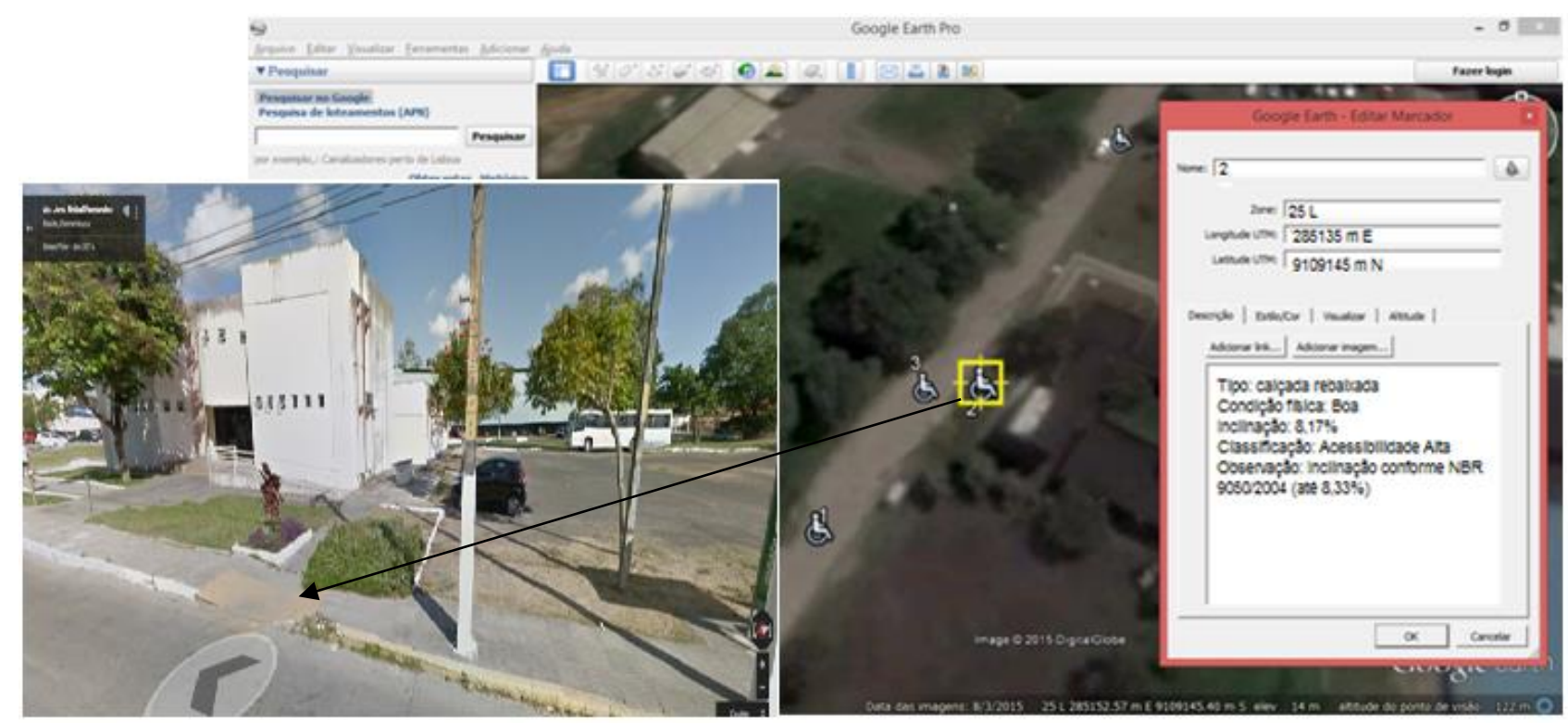

Figura 5 - Distribuição espacial dos elementos físicos para acessibilidade no programa Google Earth Pro e Street View

\section{CONSIDERAÇÕES FINAIS}

A UFPE tem se preocupado com a acessibilidade de pessoas com deficiência, mas existem locais que precisam de melhorias quanto às condições físicas das calçadas, rampas e calçadas rebaixadas além de sinalização indicativa de acesso, principalmente no trecho pesquisado.

É importante ressaltar que as Instituições de Ensino devem oferecer o acesso adequado a qualquer cidadão (aluno, funcionários e público em geral) com problemas de locomoção nos espaços.

Diante dos resultados apresentados, o uso de imagens de sensoriamento remoto associados aos dados de campo apresentaram uma boa solução para a localização das rampas e calçadas rebaixadas no trecho pesquisado.

A resolução espacial das imagens disponíveis no Google Earth Pro não é adequada para visualização das variáveis estudadas para acessibilidade e mobilidade em espaços urbanos, recomenda-se a utilização de imagens ortorretificadas com altas resoluções espaciais. Porém pode ser um auxílio a priori na visualização e planejamento do trajeto para cadeirantes. Além disso, pode servir para a prefeitura da cidade universitária na atualização e melhoria de acessos.

Recomenda-se o uso dos dados da pesquisa em aplicativos colaborativos e mapas OpenSource como OpenStreetMap, WellMap e outros, como ferramenta tecnológica para oferecer mapas de acessibilidade e auxiliar na mobilidade de pessoas em cadeiras de rodas.

A metodologia pode ser utilizada e adaptada para análise e identificação dos elementos do meio urbano específicos para as demais deficiências.

\section{AGRADECIMENTOS}

Ao Programa de Pós-Graduação em Ciências Geodésicas e Tecnologias da Geoinformação da Universidade Federal de Pernambuco pelo material de campo utilizado, ao Instituto Federal de Educação, Ciência e Tecnologia do
Piauí do qual a primeira autora faz parte. A CAPES pelas bolsas de mestrado da segunda e terceira autora.

\section{REFERÊNCIAS BIBLIOGRÁFICAS}

AGUIAR, F. de O.; SILVA, A. N. R. da; RAMOS, R. A. R., YUASSA, V. N. Avaliação da Mobilidade em Espaços Urbanos com Deficiências para Pessoas com Dificuldade de Locomoção. In: CONGRESSO LUSO BRASILEIRO PARA O PLANEJAMENTO URBANO, REGIONAL, INTEGRADO E SUSTENTÁVEL, 3., 2008, Santos. Anais... Santos, 2008.

ARAÚJO, M. P. S. de. Modelagem de dados espaciais na acessibilidade de deficientes físicos. 2005. $110 \mathrm{f}$. Dissertação (Mestrado) - Universidade Federal de Pernambuco, Recife, 2005.

ASSOCIAÇÃO BRASILEIRA DE NORMAS TÉCNICAS. NBR 9050: Acessibilidade de pessoas portadoras de deficiências a edificações, espaço, mobiliário e equipamentos urbanos. Rio de Janeiro: ABNT, 1994.

CARDOSO, R. S. C.de P. Desenvolvimento de uma Base Teórica para Aplicações em Mapas de Acessibilidade Para O Auxílio no Deslocamento de Pessoas com Mobilidade Reduzida. Centro de Ciências Matemáticas e da Natureza. Instituto de Geociências. Departamento de Geografia. UFRJ. 2015.

CARDOSO, R. S. C. de P., ALVES, S. L. P., DIONISIO, P. M., SOUZA B. C. P., MENEZES P. M. L. Mapas de acessibilidade como uma ferramenta de auxílio no deslocamento de pessoas com mobilidade reduzida. IN: XXVI CONGRESSO BRASILEIRO DE CARTOGRAFIA, 2014, Gramado. Anais... Gramado, 2014. 
GOODCHILD, M. F. The use cases of digital earth, International Journal of Digital Earth. 2008. 1:1, p.31-42.

GOODCHILD, M. F. The future of Digital Earth, Annals of GIS. 2012. 18:2, p. 93-98.

JENSEN, J.R. Sensoriamento remoto do ambiente: uma perspectiva em recursos terrestres. Tradução: EPIPHANIO, J.C.N. (coordenador)...[et al.]. São José dos Campos: Parêntese, 2009.

LEITE, N. B. F. Expansão urbana e seus efeitos sobre a mobilidade e acessibilidade avaliada com o auxílio dos sistemas de informação geográfica (SIG) em Teresina - PI. 2013. 162 f. Tese (Doutorado) - Universidade Federal de Minas Gerais: Instituto de Geociências, Belo Horizonte. 2013.
LONGLEY, P. A; GOODCHILD, M. F.; MAGUIRE, D. J.; RHIND, D. W. Geographical Information Systems: Principles, Techniques, Management and Applications. 2nd Edition. Wiley. 2013. URL:

http://www.geos.ed.ac.uk/ gisteac/gis_book_abridged/ . Acessado em 20/08/2016.

VASCONCELLOS, E. A. Transporte urbano, espaço e equidade - Análise das políticas públicas. São Paulo: Annablume, 2001. 218 p.

VASQUES, A. R. Geotecnologias nos estudos sobre brownfields: identificação de brownfields em imagens de alta resolução espacial $e$ anáise da dinâmica da refuncionalização de antigas areas fabris em São Paulo. 2009. 245 f. Tese (Doutorado) - Faculdade de Filosofia, letras e Ciências Humanas da Universidade de São Paulo, São Paulo. 2009. 